\title{
COMPACT ENDOMORPHISMS AND CLOSED IDEALS IN BANACH ALGEBRAS
}

\author{
SANDY GRABINER
}

\begin{abstract}
Every infinite-dimensional Banach algebra with a nonzero compact endomorphism has a proper closed nonzero two-sided ideal. When the algebra is commutative, the ideal is also an ideal in the multiplier algebra.
\end{abstract}

Suppose that $A$ is a Banach algebra with a compact nonzero endomorphism. We show that $A$ has a proper closed nonzero two-sided ideal, and we prove a slightly stronger result when $A$ is commutative. There are many Banach algebras which have compact endomorphisms [5]; this can even happen for commutative radical integral domains [3, §5]. On the other hand, some primitive Banach algebras, such as the algebra of compact operators on Hilbert space, have no nonzero two-sided ideals. Our results show that algebras with no nonzero closed ideals can have no compact nonzero endomorphisms.

Our proofs involve applying extensions, which we developed in [4], of Lomonosov's invariant subspace theorems. Apparently the best that one can do with the original versions of Lomonosov's theorem [6,7] is to show that if $A$ has a compact left or right multiplication operator, then it has both left and right nonzero closed ideals. Using very different methods, Esterle [1] has recently made significant contributions to the study of the problem of whether every radical Banach algebra has a nonzero closed ideal.

THEOREM 1. If the infinite-dimensional Banach algebra $A$ has a compact nonzero endomorphism $\phi$, then $A$ has a proper closed nonzero ideal.

Proof. Let $A^{\#}$ be the Banach algebra formed by adjoining an identity to $A$ if $A$ has no identity, and let $A^{\sharp}=A$ if $A$ has an identity. For each $a$ in $A^{\sharp}$, let $L_{a}$ be the left multiplication operator $L_{a} x=a x$, and let $\mathscr{L}$ be the algebra of all left multiplication operators $L_{a}$ on $A$ for $a$ in $A^{\#}$. Similarly, let $\mathscr{R}$ be the algebra of right multiplication operators. To show that $A$ has a proper closed ideal, we show that $\mathscr{L}$ and $\mathscr{R}$ have a common proper invariant subspace. We do this by showing that the algebras $\mathscr{L}$ and $\mathscr{R}$ satisfy the hypotheses of [4, Theorem (3.2), p. 849].

The map $a \rightarrow L_{a}$ is a continuous linear transformation from $A^{\sharp}$ to the algebra of bounded operators on $A$. Hence $\mathscr{L}$ is an operator range algebra in the sense of [4, p. 845] (cf. [2, §3]). Since $\phi$ is a homomorphism, we have $\phi L_{a}=L_{\phi(a)} \phi$ for every $a$ in

Received by the editors December 21, 1983.

1980 Mathematics Subject Classification. Primary 46H10; Secondary 46J20, 47A15. 
$A^{\sharp}$, so that $\phi \mathscr{L} \subseteq \mathscr{L} \phi$. Similarly, $\mathscr{R}$ is an operator range algebra with identity and $\phi \mathscr{R}$ $\subseteq \mathscr{R} \phi$, so that $\phi \mathscr{L} \mathscr{R} \subseteq \mathscr{L} \mathscr{R} \phi$. Finally, $\mathscr{L} \mathscr{R}=\mathscr{R} \mathscr{L}$, so it follows from [4, Theorem (3.2), p. 849] that $\mathscr{L}$ and $\mathscr{R}$ have a common invariant subspace which must be a closed nonzero proper ideal in $A$.

THEOREM 2. Suppose that $A$ is a commutative Banach algebra which contains at least one element a for which the map $x \rightarrow$ ax is not multiplication by a scalar. If $A$ has a compact nonzero endomorphism $\phi$, then there is a proper closed nonzero subspace of $A$ which is an ideal in the multiplier algebra of $A$.

Proof. As in the proof of Theorem 1 , we let $\mathscr{L}$ be the operator range algebra of left multiplications by elements of $A^{\sharp}$, and we have $\phi \mathscr{L} \subseteq \mathscr{L} \phi$. It then follows from [4, Theorem (3.1), p. 848], or [2, Theorem 6, p. 61] that the commutant of $\mathscr{L}$ has an invariant subspace. Since the commutant of $\mathscr{L}$ is the multiplier algebra of $A$, the theorem is proved.

If the algebra $A$ in Theorem 2 is not commutative, we can use [4, Theorem (3.2), p. 849] to conclude that $A$ has a proper nonzero closed subspace which is invariant under all operators which are simultaneously right and left multipliers of $A$. However, such a subspace need not even be an ideal of $A$, since we can guarantee only that it is closed under multiplication by central elements of $A$.

\section{REFERENCES}

1. J. Esterle, Quasimultipliers, representations of $H^{\infty}$, and the closed ideal problem, Radical Banach Algebras and Automatic Continuity (J. Bachar et al., eds.), Lecture Notes in Math., vol. 975, SpringerVerlag, Berlin, 1983, pp. 66-162.

2. C. K. Fong et al.,Extensions of Lomonosov's invariant subspace theorem, Acta Sci. Math. (Szeged) 41 (1979), 55-62.

3. S. Grabiner, Derivations and automorphisms of Banach algebras of power series, Mem. Amer. Math. Soc. No. 146 (1974).

4. __ Operator ranges and invariant subspaces, Indiana Univ. Math. J. 28 (1979), 845-857.

5. H. Kamowitz, Compact endomorphisms of Banach algebras, Pacific J. Math. 89 (1980), 313-325.

6. V. Lomonosov, Invariant subspaces for operators which commute with a completely continuous operator, Funct. Anal. Appl. 7 (1973), 213-214.

7. C. Pearcy and A. L. Shields, $A$ survey of the Lomonosov technique in the theory of invariant subspaces, Topics in Operator Theory (C. Pearcy, ed.), Math. Surveys, vol. 13, Amer. Math. Soc., Providence, R. I., 1974.

Department of Mathematics, Pomona College, Claremont, California 91711 